\title{
SMALL-INTEGER RATIOS PREDOMINATE THROUGHOUT THE WORLD'S MUSICAL SCALES
}

\author{
Gakuto Chiba ${ }^{1}$, Meng-Jou Ho ${ }^{1}$, Shoichiro Sato ${ }^{1}$, Jiei Kuroyanagi ${ }^{1}$, Joren Six ${ }^{2}$, Peter Pfordresher ${ }^{3}$, \\ Adam Tierney ${ }^{4}$, Shinya Fujii ${ }^{1}$, Patrick E. Savage*1 \\ ${ }^{1}$ Keio University, Japan, ${ }^{2}$ Ghent University, Belgium, ${ }^{3}$ University at Buffalo, NY, USA, ${ }^{4}$ Birbeck, University of London, \\ UK \\ *Correspondence to: psavage@sfc.keio.ac.jp
}

\begin{abstract}
The structure of musical scales has been proposed to reflect universal bioacoustic principles based on simple integer ratios. However, some researchers who have studied tuning in small samples of non-Western cultures have argued that such ratios are instead specific to Western music. To address this debate, we algorithmically analyzed and cross-culturally compared scale tunings within a global sample of 124 music recordings, including both instrumental and vocal music. Although we found great cross-cultural diversity in most scale degrees, we also found a strong cross-cultural tendency to include the simplest possible integer ratios within the octave (3:2 ratio [perfect 5th] and 4:3 ratio [perfect 4th]). This suggests that cultural diversity in musical scales is not without limit, but is constrained by general bioacoustic principles, such as harmonic consonance in group performance, that may shed light on the evolution of human music.
\end{abstract}

\section{BACKGROUND}

Music, like language, is a human universal found in every known culture throughout world history. Although music takes many different forms cross-culturally, scientists have identified numerous "statistical universals" that predominate in most - but not all - of the world's music [10]. One hypothesis to explain musical universals proposes that the striking cross-cultural convergence of the structure of musical scales (e.g., pentatonic scales; cf. Fig. 2) may reflect universal bioacoustic principles based on simple integer ratios, because such ratios maximize the overlap among harmonic frequency spectra and thus sound more consonant [1]. However, cross-cultural analyses of both instrument tunings [2] and perceptions of consonance [6] in small samples of non-Western cultures concluded that no such cross-cultural preferences for simple-integer ratios exist. This debate remains unresolved in part because of a lack of objective global data on scale tunings. Our goal was to address this lack of data by taking advantage of new algorithms for automatic scale tuning analysis [12]. Based on the integer ratio hypothesis and previous preliminary studies $[3,4,9]$, we predicted that the simplest ratios such as the perfect $5^{\text {th }}(3: 2)$ and perfect $4^{\text {th }}$ (4:3) would predominate cross-culturally.

\section{METHOD}

\subsection{Music Sample}

For this analysis, a subset of all available monophonic recordings $(\mathrm{n}=124)$ was selected from the full 304 companion recordings to the Garland Encyclopedia of World Music [7] that were previously analyzed manually [10]. Polyphonic recordings or recordings with loud background noise for which automatic pitch estimation could not be performed accurately were excluded.

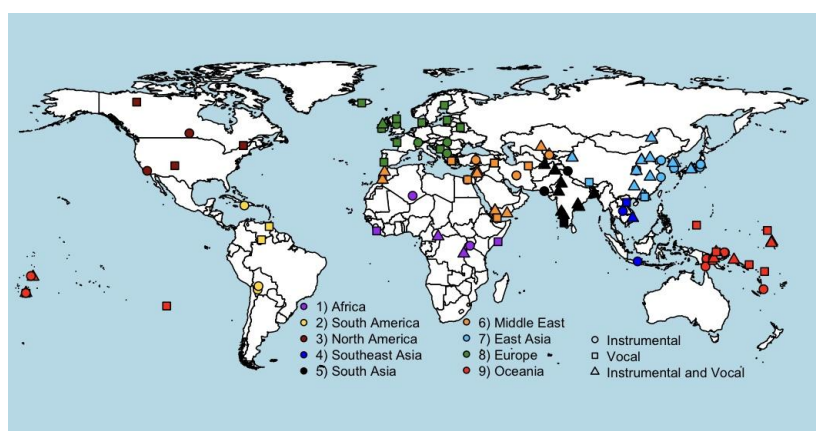

Figure 1. Map of the 124-recording sample labeled according to geographic region and instrumentation.

\begin{tabular}{|c|c|c|c|c|}
\hline & Instrumental & Vocal & Mixed & TOTAL \\
\hline Africa & 2 & 2 & 2 & 6 \\
\hline South America & 3 & 2 & 0 & 5 \\
\hline North America & 2 & 4 & 0 & 6 \\
\hline Southeast Asia & 2 & 1 & 1 & 4 \\
\hline South Asia & 2 & 1 & 9 & 12 \\
\hline
\end{tabular}




\begin{tabular}{|c|c|c|c|c|}
\hline Middle East & 5 & 8 & 10 & 23 \\
\hline East Asia & 11 & 2 & 16 & 29 \\
\hline Europe & 4 & 10 & 1 & 15 \\
\hline Oceania & 7 & 9 & 8 & 24 \\
\hline TOTAL & 38 & 39 & 47 & $\mathbf{1 2 4}$ \\
\hline
\end{tabular}

Table 1. Distribution of instrumental and vocal music recordings across the nine regions.

\subsection{Pitch Class Histogram}

We used Tarsos [12] to analyze and compare tunings because, unlike most MIR algorithms that are based on scale models incorporating Western 12-tone equal temperament, Tarsos was designed explicitly for automatic analysis of any music from around the world without imposing such culture-specific theories. Tarsos first extracts the pitch histogram, then collapses this pitch histogram across octaves to create a pitch class histogram, expressed in cents [2] ranging from 0-1200. We used Tarsos's default YIN pitch estimation algorithm.

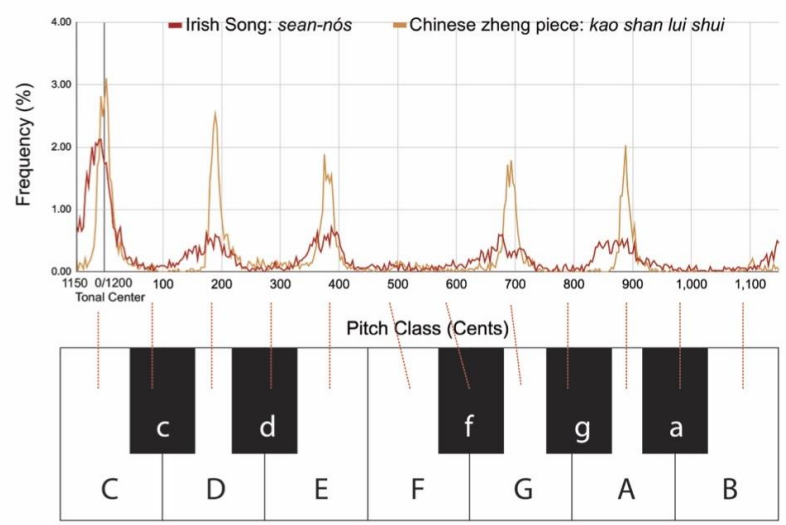

Figure 2. A traditional Irish song and a Chinese instrumental piece demonstrate similar pentatonic scales. The $\mathrm{x}$-axis represents pitch class (octave-equalized) in cents, whereas the $y$-axis represents frequency of occurrence for each pitch class.

\subsection{Normalization to the Tonal Center}

In order to meaningfully compare scales between different songs that use different absolute pitches, previous researchers have attempted to normalized each song to a shared tonal center by setting the pitch class of its final note or its most frequent note to 0 cents. However, it was unclear whether this choice of note for normalization affected the results. To empirically test this, we ran the analyses twice, once normalizing to the final note, and again normalizing to the most frequent note.

\section{RESULTS}

\subsection{Final note vs. most frequent note}

Our analysis of average pitch class histograms across all 124 recordings shows that there is little difference between normalizing to the most frequent note or to the final note (Fig. 3). Both methods revealed strong peaks at the perfect 5 th (3:2 ratio, $\sim 700$ cents) and perfect 4 th (4:3 ratio, $\sim 500$ cents), as well as a weaker but clear peak at the major 2nd (9:8 ratio, 200 cents). Normalizing to the final note gives a slightly sharper perfect 5 th peak than using the most frequent note, but otherwise the average pitchclass histograms are practically identical. Given the difficulty in determining final notes in music involving fade-outs or recordings without discrete pitches, we thus decided to normalize using the most frequent note for all subsequent sub-sample analyses.

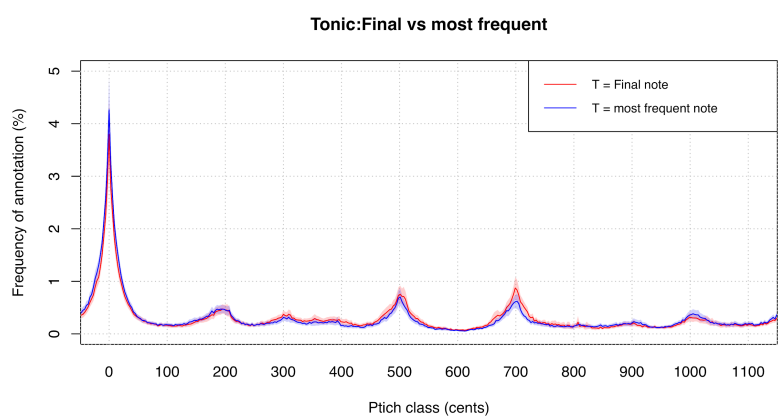

Figure 3. Average pitch class histograms across all recordings $(n=124)$ normalizing to the most frequent note (blue) and final note (red). The $\mathrm{x}$-axis begins and ends at 1150 cents in order to show the distribution around the tonal center (set to 0 cents).

\subsection{Instrumental vs. vocal music}

Analyses breaking down the sample into subsamples based on instrumentation confirm that the same three intervals perfect 5 th, perfect 4 th, and major 2nd - consistently predominate for both vocal and instrumental music (Fig. 5). However, the peaks s tend to be much less precise for vocal than instrumental music, with mixed vocal and instrumental music showing levels of precision intermediate between pure vocal and pure instrumental music. This is consistent with previous research showing that vocal music is much less precise than instrumental [8] 

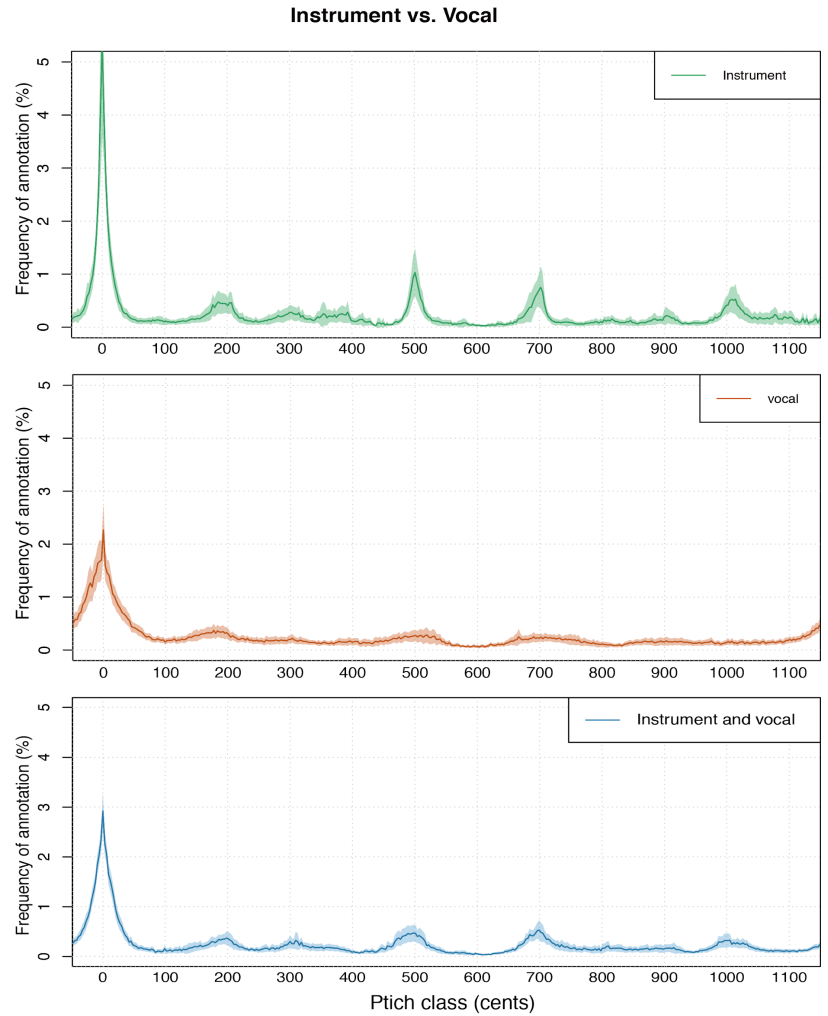

Figure 5. Average pitch class histograms for instrumental (green, $\mathrm{n}=38$ ) vocal (orange, $\mathrm{n}=39$ ), and mixed (blue, $\mathrm{n}=47$ ) sub-samples.

\subsection{Regional analyses}

Dividing the 124 songs into the 9 regions pre-classified by the Garland editors shows that the peaks at perfect 4th and 5 th are clear in almost every region. The exception appears to be Oceania, where music tends to have narrow ranges that may not even extend as far as a perfect 5th [5].
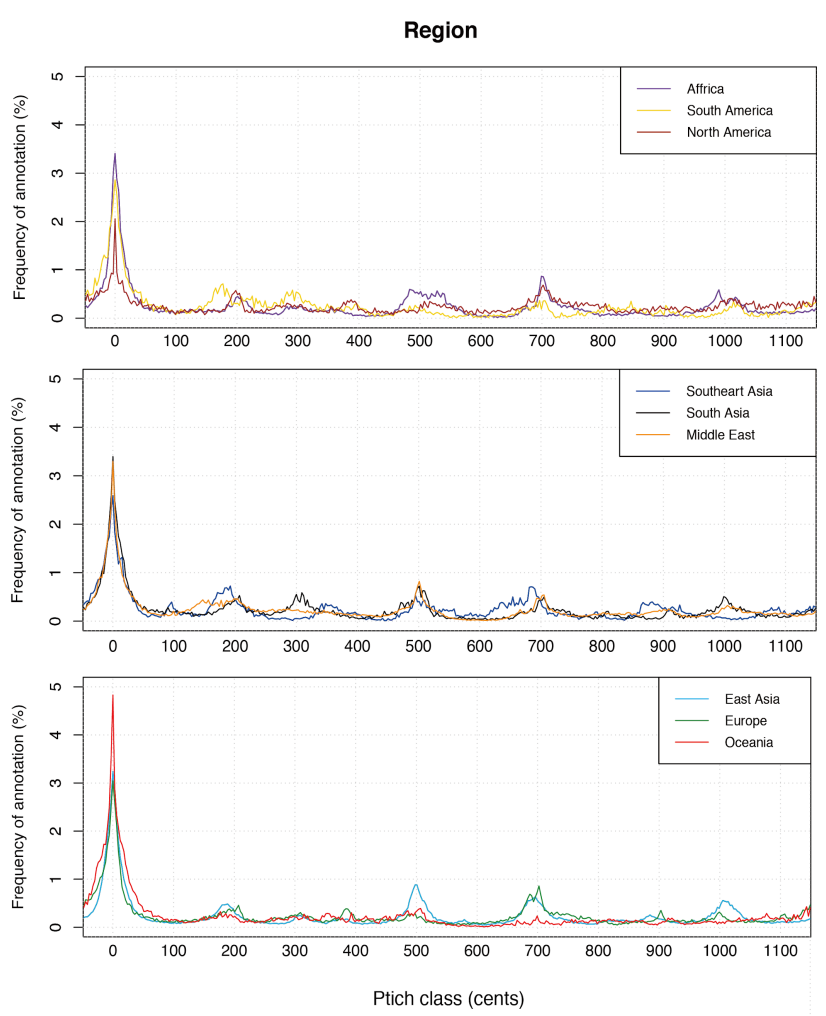

Figure 6. Average pitch class histograms for each of the 9 regions shown in Fig. 1 and Table 1.

\section{DISCUSSION}

Our automatic analyses of scale tunings from diverse music recordings from around the world show that smallinteger ratios - particularly the perfect 5th (3:2 ratio) and perfect 4 th (4:3 ratio) consistently predominate throughout the world's vocal and instrumental music. The fact that the simplest possible ratios within the octave were the most universal supports the integer ratio hypothesis based on perceptual consonance [1]. However, the fact that average vocal tunings were much less precise than instrumental tunings, that Oceanian music had restricted ranges without a clearly predominant perfect 5 th, and separate data showing differences in scale tuning precision between children's and adult music [9] could be argued to provide support for the competing vocal mistuning hypothesis, which proposes a motor origin of scale structure [8]. A sensorimotor hypothesis that combines these two theories may provide a more powerful theory for explaining unity and diversity in the world's scales $[8,9]$

\section{FUTURE WORK}

Currently, our analysis assumes octave equivalence by collapsing pitch histograms into pitch class histograms. However, in the future we could empirically test for the universality of octave equivalence, especially since the integer ratio hypothesis predicts that the octave (2:1 ratio) should be the most universal interval of all.

Currently, our analyses requires manual screening of recordings that are not analyzable using Tarsos and manual normalization to a tonal center. Automating this process would allow us to potentially expand this preliminary 
analysis to include thousands or even millions of recordings. Comparing such automated musical analysis against samples of speech and birdsong should allow us to determine whether aspects of musical scales are specific to human music $[4,11,13]$. Meanwhile, comparison against cross-cultural data for perceptions of consonance should help to reveal the causal mechanisms underlying crosscultural musical regularities. This may in turn help to us understand the evolutionary origins of music and its importance in human cultures throughout the world.

\section{AUTHOR CONTRIBUTIONS}

P.E.S., S.F., A.T., J.S., and P.Q.P. designed the study; C.G., M.-J.H., S.S., and J.K., analysed the data, supervised by P.E.S.; C.G., M.-J.H., and P.E.S. drafted the manuscript.

\section{ACKNOWLEDGMENTS}

This work was supported by a Shonan Fujisawa Gakkai Travel Award to M.-J.H., and a Japan Society for the Promotion of Science Grant-in-Aid for Young Scientists, a Keio Research Institute at SFC Startup Grant, and a Keio Gijuku Academic Development Fund Individual Grant to P.E.S. We thank Steven Brown for comments on earlier versions of this manuscript. This is an expanded version of work presented at the Late-Breaking Demo session of the 2018 International Society for Music Information Retrieval (ISMIR) conference [3].

\section{REFERENCES}

[1] D. L. Bowling, and D, Purves. "A biological rationale for musical consonance," Proceedings of the National Academy of Sciences of the U.S. A., vol.112, no. 36, 2015

[2] A. J. Ellis, "On the musical scales of various nations," Journal of the Society of Arts, vol.23, no.1688, pp. 435-527, 1885.

[3] M.-J. Ho, S. Sato, J. Kuroyanagi, J. Six, S. Brown, S. Fujii, and P. E. Savage, "Automatic analysis of global music recordings suggests scale tuning universals," in Extended abstracts for the Late-Breaking Demo Session of the 19th International Society for Music Information Retrieval Conference (ISMIR 2018), 2018.

[4] J. Kuroyanagi et al., "Automatic comparison of human music, speech, and bird song suggests uniqueness of human scales," in Proceedings of the 9th International Workshop on Folk Music Analysis (FMA2019), $2019 . \quad$ Preprint: https://doi.org/10.31234/osf.io/zpv5w

[5] A. Lomax, Ed., Folk song style and culture. Washington, DC: American Association for the Advancement of Science, 1968.

[6] J. H. McDermott, A. F. Schultz, E. A. Undurraga, and R. A. Godoy, "Indifference to dissonance in native
Amazonians reveals cultural variation in music perception," Nature, vol. 535, pp. 547-550, 2016.

[7] B. Nettl, R. Stone, J. Porter, and T. Rice, Eds., The Garland encyclopedia of world music [10 volumes; 9 CDs]. New York: Garland Pub., 1998-2002.

[8] P. Q. Pfordresher and S. Brown, "Vocal mistuning reveals the origin of musical scales," Journal of Cognitive Psychology, vol. 29, no. 1, pp. 35-52, 2017.

[9] S. Sato, J. Six, P. Pfordresher, S. Fujii, and P. E. Savage, "Automatic comparison of global children's and adult songs supports a sensorimotor hypothesis for the origin of musical scales," in Proceedings of the 9th International Workshop on Folk Music Analysis (FMA2019), 2019. Preprint: https://doi.org/10.31234/osf.io/kt7py

[10] P. E. Savage, S. Brown, E. Sakai, \& T. E. Currie, "Statistical Universals Reveal the Structures and Functions of Human Music," Proceedings of the National Academy of Sciences of the U.S.A., vol.112, no.29, pp. 8987-8992 2015.

[11] P. E. Savage, A. T. Tierney, and A. D. Patel, "Global music recordings support the motor constraint hypothesis for human and avian song contour," Music Perception, vol. 34, no. 3, pp. 327-334, 2017.

[12] J. Six, O. Cornelis, and M. Leman, "Tarsos: A modular platform for precise pitch analysis of Western and non-Western music," Journal of New Music Research, vol. 42, no. 2., pp.113-129, 2013.

[13] A. T. Tierney, F. A. Russo, and A. D. Patel, "The motor origins of human and avian song structure," Proceedings of the National Academy of Sciences of the U. S. A., vol. 108, no. 37, pp. 15510-15515, 2011. 